\title{
Globe
}

Revue internationale d'études québécoises

\section{Vers une histoire de la religion en dehors du religieux? De quelques publications québécoises récentes portant sur l'histoire de l'église catholique}

Jean-Philippe Warren

Volume 11, numéro 1, 2008

URI : https://id.erudit.org/iderudit/1000502ar

DOI : https://doi.org/10.7202/1000502ar

Aller au sommaire du numéro

Éditeur(s)

Globe, Revue internationale d'études québécoises

ISSN

1481-5869 (imprimé)

1923-8231 (numérique)

Découvrir la revue

Citer cette note

Warren, J.-P. (2008). Vers une histoire de la religion en dehors du religieux? De quelques publications québécoises récentes portant sur l'histoire de l'église catholique. Globe, 11(1), 255-268. https://doi.org/10.7202/1000502ar d'utilisation que vous pouvez consulter en ligne. 


\section{VERS UNE HISTOIRE}

\section{DE LA RELIGION EN}

\section{DEHORS DU RELIGIEUX?}

\section{DE QUELQUES \\ PUBLICATIONS}

\section{QUÉBÉCOISES RÉCENTES}

\section{PORTANT SUR L'HISTOIRE DE L'ÉGLISE CATHOLIQUE}

\section{JEAN-PHILIPPE WARREN \\ Université Concordia}

Ceux-là qui, il y a quelques années à peine, décriaient le faible intérêt des chercheurs québécois pour l'histoire de l'Église catholique, reléguée dans l'ombre des études plus "sérieuses" sur le libéralisme ou le nationalisme, peuvent se réjouir. La récolte des dernières saisons est abondante. Quoiqu'il faut reconnaître l'existence d'un corpus de recherche qui n’a pas attendu, pour se constituer, l'arrivée de la jeune génération, il me semble que des initiatives comme la fondation de la revue Mens témoignent d'une volonté de reprendre à nouveau frais les vieux débats sur la place et le rôle de l'Église. Les multiples facettes de la vie religieuse tombent sous la loupe de nombreux jeunes historiens qui publient leurs travaux de maîtrise ou de doctorat, ou les premiers fruits de leurs recherches subventionnées. Cette note critique vise à faire connaître l'intention derrière leurs contributions et les principaux axes de leurs réflexions. Elle n'entend pas, il va sans dire, épuiser la richesse des parcours individuels ni l'originalité des perspectives adoptées par les uns et les autres. L'objectif est beaucoup plus 
humble: d'une part, formuler quelques hypothèses qui nous permettent de mieux saisir l'intérêt pour le catholicisme canadien-français et, d'autre part, cerner la spécificité du regard qui, depuis quatre ou cinq ans, est jeté sur celui-ci. Tentant de répondre à ces deux questions distinctes, cette note critique est divisée en deux parties autonomes: pourquoi les jeunes chercheurs s'intéressent-ils au fait religieux en histoire, et comment s'y intéressent-ils?

\section{TROIS MOTIVATIONS PRINCIPALES}

À l'extérieur de la province, les Canadiens anglais n'ont pas attendu ce regain d'intérêt pour publier des travaux substantiels qui incorporent, dans leur lecture du passé canadien-français, la dimension religieuse. Parmi une pléiade de publications, les ouvrages de Michael Oliver, Michael Behiels, Joseph Levitt et Susan Mann (Trofimenkoff) viennent spontanément à l'esprit. Il ne faisait guère de doute, pour eux, qu'un des traits distinctifs du Québec, c'était d'être une province où les prêtres occupaient une position privilégiée. Intrigués par le nationalisme de leurs concitoyens de langue française, its le liaient à un certain conservatisme, lui-même nourri par la tradition cléricale, même s'ils se refusaient à condamner $a$ priori ce patriotisme. Il y avait là pour eux un simple fait à comprendre. En prenant refuge dans le giron de l'Église, les Canadiens français avaient peut-être, selon ces auteurs, tourné le dos à l'Amérique et au progrès, mais ils développaient en même temps un univers de valeurs qui ne manquait pas de charmes et d'attraits, sinon même de vérité et de justice. Du côté des auteurs de langue française, Fernand Dumont, Nicole Laurin, Jean Hamelin et combien d'àutres ont également creusé la question depuis trente ou quarante ans. Ces derniers ne croyaient pas que l'Église avait constitué un corps uniment réactionnaire, mais, au contraire, ils envisageaient cette institution comme une réalité complexe, dont certains éléments avaient même pu jouer un rôle avant-gardiste. AndréJ. Bélanger écrivait, par exemple, que "la distanciation d'avec le clergé" s'était réalisée "à l'intérieur même de l'encadrement ecclésial", et il allait jusqu'à écrire que «la rupture pour une bonne partie de l'intelligentsia québécoise prend là sa source ${ }^{1}$ ". Poursuivant dans cette veine, Michael Gauvreau a fait paraître, en 2005 , un ouvrage qui, sur 500 pages, s'attache à réinventer la roue, mais avec une telle érudition et une telle finesse du propos

$$
++4
$$

1. André-J. BÉLANGER, Ruptures et constantes. Quatre adéologres du Quebec en éclatement. La Relève, La JEC, Cité libre, Parci pris, Monuréal, Hurtubise HMH, 1977, p. 36. 
qu'on en oublie presque que tout cela avait déjà été dit en français, des années auparavant ${ }^{2}$.

Qu'en est-il du côté des jeunes historiens québécois de langue française? Trois motifs expliquent, selon moi, l'intérêt renouvelé des jeunes chercheurs francophones pour l'histoire de l'Église catholique. La première raison, la plus directe, c'est le choix de la période analysée. Après avoir écrit de vastes monographies sur la Nouvelle-France, sur ses héros et ses combats, après avoir fait paraître des livres sur le XIX siècle et le tournant $\mathrm{du} X \mathrm{XX}^{\mathrm{e}}$ siècle, ce n'est que récemment, après que le reste du champ ait été en quelque sorte occupé par des chercheurs plus établis, que des thésards ont fait le choix "en masse" d'interroger une époque encore plus récente, celle de l'entre-deuxguerres et de l'immédiat après-guerre. Lucie Piché, par exemple, a publié une thèse sur les associations de jeunesse catholique spécialisées pendant la parenthèse de la Grande noirceur (1931-1966). Elle y a découvert non seulement un foisonnement d'idées et de débats, et une société bien moins sombre que le préjugé dominant le suppose, mais aussi un éléphant incontournable: l'Eglise catholique, qui est alors à peu près partout dans la société civile (exception faite du monde des affaires). Même constat du côté de la thèse de Nicole Neatby consacrée aux étudiants de l'Université de Montréal durant la période duplessiste: les écoles sont catholiques, les coopératives sont catholiques, les associations de loisirs populaires sont catholiques, les hôpitaux sont catholiques et tutti quanti. Certes, il existe une pléiade de domaines qui ne tombent pas sous la chape de l'institution cléricale: ni La Presse, ni le Ouimetoscope, ni l'État provincial, ni Eaton, ni les syndicats internationaux, ni même Santa Claus ne sont catholiques. II n'en reste pas moins que ce qui colore cette période, ce qui lui donne sa cohérence, ce qui assure son encadrement social et sa représentation collective, c'est avant tout la religion romaine.

La seconde raison pour laquelle l'Église est l'objet d'un regain d'attention, c'est que, longtemps négligé, le champ de l'histoire intellectuelle est mis à l'honneur depuis quelque temps. Quand le paradigme de l'histoire sociale dominait les départements universitaires, et qu'il n'y en avait que pour les chiffres et les statistiques du développement socio-économique, l'Église pâtissait de ce parti pris. Dans la perspective de la modernisation de la société québécoise, les effectifs cléricaux ne font, en effet, pas le poids. Que valent

$$
+4
$$

2. Michael Gauvreau, The Catholic Origins of Quebec's Quiet Revolution, 1931-1970, Montréal/Kingston, McGillQueen's University Press, 2005. 
une poignée de curés qui s'acharnent à publier d'obscures revues prosélytes devant les avancées des médias de masse? Que dire des discours autour de la "revanche des berceaux", alors que les taux de natalité déclinent irrésistiblement? Pourquoi se pencher sur l'entreprise des coopératives catholiques, alors que le $\mathrm{XX}^{e}$ siècle est sans conteste celui de la grande industrie? Dans la perspective de l'histoire sociale, l'Église tient peu de place, sinon comme repoussoir à ce qui se trame sans elle (même chez Jean-Marie Fecteau, où elle est en partie réhabilitée comme complice du libéralisme). Mais dans la perspective d'une histoire intellectuelle, alors le petit Devoir devient incontournable, Papin Archambault est réhabilité, les très marginales idées corporatistes se retrouvent au cœur des analyses, les numéros plutôt juvéniles de la revue $L a$ Relève sont feuilletés avec intérêt. Le monde des idées conservatrices s'anime, les cimetières où reposent en paix les André Laurendeau et les Lionel Groulx retentissent des cris des débats d'autrefois. Il ne s'agit plus de retracer l'évolution des structures sociales, mais de saisir les mutations des discours, de refaire la généalogie des idées. Or, comme ces discours et ces idées sont fortement habités par des valeurs, sinon des injonctions tirées de la tradition religieuse, les encycliques papales (qui, en somme, n'ont eu qu'un impact discret sur la planification urbaine, l'exploitation forestière, la transformation des universités en centres de recherche ou la montée de la société de consommation) sont intégrées dans l'analyse. Ces encycliques ont, en effet, provoqué moult remous dans les cercles intellectuels, voire créé l'espace pour l'engagement des religieux et des laïcs dans le grand combat qui opposait les "idées vraies» et les "idées fausses".

Inspirés par la doctrine sociale de l'Église énoncée par l'encyclique Rerum novarum, les intellectuels canadiens-français se donnent une nouvelle fonction au tournant du $\mathrm{XX}^{\mathrm{e}}$ siècle: celle de l'étude méthodique des problèmes des Canadiens français, dont les solutions sont proposées sur la place publique ${ }^{3}$.

La dernière motivation qui permet de comprendre l'enthousiasme des jeunes chercheurs, c'est une certaine empathie pour le conservatisme. Cette empathie permet de comprendre en partie pourquoi la conscience collective actuelle se reconnaît davantage dans les idées et les idéaux du Canada français. Jacques Beauchemin a sans doute donné ses lettres de

$$
++
$$

3. Pascale RYan, Penser la nation. La Ligue d'action natzonale, 1917-1960, Montréal, Leméac, 2006, p. 15. 
noblesse à cette attitude plus conciliante, lui dont l'œuvre vise justement à rescaper une part de cet héritage. Au contraire de ses prédécesseurs, qui percevaient le Canada français comme un monde réactionnaire (ce que traduit bien l'expression de Grande noirceur), Beauchemin interprète le parcours de cette collectivité de manière fort positive. La culture francophone, écrit-il dans L'Histoire en trop, plonge ses racines "dans le terreau profond de la mémoire canadienne-française». Une mémoire qui n'est pas seulement faite d'exclusions et d'oppression. On y trouve aussi, selon lui, "une expérience, vieille de deux siècles et demi, d'accueil de l'altéritét». Cette posture plus empathique, on la teconnaît également chez Dominique Foisy-Geoffroy, qui avoue avoir cherché à comprendre l'œuvre d'Esdras Minville de l'intérieur. L'historien affirme en conclusion de son essai :

La pensée minvillienne, comme le traditionalisme en général, se distingue par une conception du passé qui mérite toute notre attention. En effet, les traditionalistes entretiennent un rapport empreint de respect, de déférence polie mais non dénuée d'esprit critique envers l'histoire conçue comme riche dépôt du patrimoine humain, comme précieux héritage des ancêtres à préserver et enrichir. Les historiens québécois, comme la population en général, auraient intérêt à revenir à l'esprit d'un tel rapport plus serein au passés.

Pareille morale, où l'histoire devient le "précieux héritage des ancêtres à préserver et enrichir" ne convaincra pas l'ensemble des lecteurs, peu s'en faut. Elle semble indiquer chez certains, et c'est sur ce point que je voudrais insister, un singulier rapprochement entre la conscience historienne contemporaine et l'esprit des intellectuels de l'entre-deux-guerres.

Curieusement, nous pourrions trouver un contre-exemple facile à la présentation que nous venons de faire, puisque l'imposante thèse de doctorat de Xavier Gélinas laisse une place plutôt maigre à la religion. Dans La Droite intellectuelle québécoise et la Révolution tranquille, l'auteur a décidé d'écarter les dimensions spirituelles de son étude historique. C'est sans doute que sa période, celle de la Révolution tranquille, correspond à un moment où "l'alliance, naguère dure comme le roc, entre les idées traditionnelles, le

$$
+4
$$

4. Jacques BEAuCHEMIN, LHistoire en trop. La mauvaise conscience des souveratnistes québécois, Montréal, VLB éditeur, 2002, p. 173.

5. Domınique FoISY-GEOFFroY, Esdras Minville. Nationalsme economigue et catholicisme social au Québec durant l'entredewx-guerres, Sillery, Septentrion, 2004, p. 157. 
nationalisme et le catholicisme, s'effrite ${ }^{6}{ }^{\prime}$. Cela vient sans doute aussi d'une volonté, chez l'auteur, d'affirmer que le conservatisme politique peut être formulé en dehors des religions. Le dernier chapitre ne porte-t-il pas sur la sécularisation de la société québécoise dans les années soixante - un moment où la droite éprouve force difficultés à maintenir ses positions, une fois rompue son alliance avec «notre sainte mère l'Église» ? C'est comme si Gélinas voulait, en filigrane, nous convaincre de la validité et de la pertinence des thèses droitistes, même pour une société comme la nôtre qui a perdu son "âme" catholique, et qui ne cherche plus à réconcilier le trône et l'autel. Écoutons plutôt la conclusion : après avoir déclaré que "la droite intellectuelle n'a pas à rougir de ses propos tenus durant la Révolution tranquille ", Gélinas enchaîne en affirmant que le renouveau social est "trompeur", non seulement trompeur mais "fatal", "s'il ne s'op[ère] dans la fidélité aux points forts de l'histoire canadienne-française et à des principes jugés éternels». Pourtant, on se demandera quelle est cette fidélité aux "points forts" du passé canadien-français, si cette fidélité renonce tout à fait aux traditions spirituelles d'une nation qui s'est longtemps perçue en termes messianiques, et quels peuvent bien être des "principes jugés éternels", une fois écartée la révélation biblique? Il me semble que cet appel à la mémoire demeure trop censuré pour être reçu tel quel. Mais, appel à la mémoire il y a néanmoins.

À l'évidence, maints auteurs, dont Marie-Aimée Cliche ${ }^{7}$, ne partagent pas cette interprétation du passé canadien-français. Ces chercheurs sont, en général, un peu plus âgés. Ils voient dans le discours autoritaire des années d'entre-deux-guerres une conception plus négative de l'existence humaine. La violence faite aux enfants est un cas exemplaire, puisque les bastonnades et les châtiments infligés aux mineurs ne peuvent être rangés, pour personne, parmi les contributions méritoires des mœurs anciennes! En bref, la communauté savante tout entière ne s'est pas convertie à l'analyse des discours et au conservatisme, et encore moins à l'étude de la seule période du Canada français. Loin de là. La tendance que je crois percevoir n'est qu'une tendance. Elle fait, cependant, se rejoindre assez de gens qui, autrement, ne partagent pas les mêmes points de vues ni les mêmes conclusions (pensons seulement à Éric Bédard, Martin Meunier, Dominique Marquis, Michel

$$
+4
$$

6. Xaxier GélıNAS, La Droite intellectuelle québécoise et la Révolution tranquille, Québec, Presses de P'Université Laval, 2007 , p. 156.

7. Lire l'excellent Marie-Aimée CliCHE, Maltratter ou punir? La violence envers les enfants. 1850-1969, Montréal, Boréal, 2007. 
Bock), pour marquer les contours d'une certaine sensibilité historique ${ }^{8}$. Ainsi, Martine-Emmanuelle Lapointe, professeure de littérature, se décrit, sur le site Internet de l'Université de Montréal, comme une personne qui

s'intéresse plus particulièrement aux rapports entre histoire et mémoire dans les œuvres littéraires contemporaines, à la réception critique ainsi qu'à la question des filiations et des héritages intellectuels dans le roman anglophone et francophone du Québec?.

Une telle description serait difficilement imaginable pour ceux qui appartiennent au courant de l'histoire sociale.

\section{LE DÉBAT AUTOUR DE LA MODERNITÉ RELIGIEUSE}

Demeurant à l'intérieur du cercle d'une histoire des idées, les travaux parus dernièrement font une large place aux combats des catholiques canadiensfrançais. Comme ces études portent sur des représentations et des discours, les jeunes historiens n'échappent pas à la question de leur plus ou moins forte modernité. C'est encore et toujours cette question qu'ils trimballent avec eux, bien que ce débat récurrent des "Anciens et des Modernes" trouve à s'exprimer de manière légèrement différente. Si, pour reprendre les carégories de Ronald Rudin, l'École modernisatrice avait refoulé ces paroles dans le grand trou de la pensée réactionnaire, et si l'École normalisatrice avait voulu démontrer que, au-delà des paroles, les gestes des Canadiens français étaient "modernes", il semble que maints jeunes historiens veulent maintenant dénicher la modernité jusque dans les discours de jadis. "Les idées progressistes, déclare Suzanne Clavette, dominaient chez les catholiques sociaux ${ }^{10}$." Quant à Dominique Marquis, son ouvrage sur L'Action catholique cherche aussi à convaincre le lecteur que l'Église catholique fut bien moins figée qu'on le croit d'ordinaire. Il s'agit par conséquent d'écrire une histoire des brèches, comme le proposait il y a longtemps Yvan Lamonde, et de dénicher, avant la Révolution tranquille, des foyers de contestation ou d'adaptation sociale qui annoncent les réformes à venir (Paul-Émile Borduas en arts, Jean-Charles Harvey en journalisme, Saint-Denys Garneau en

$$
++
$$

8. Sréphane KELLY [dir.], Les Idées mènent ke Québec. Essais sur une sensibilité historqque, Sainte-Foy, Presses de l'Université Laval, 2002. Lire aussi Lucille BEAUDRY et Marc CHEVRIER [ed.], Une pensée liberale, crinque ou conservatnce? Actualité de Hannah Arendt, d'Emmanuel Mounter et de George Grant pour le Québec d'aujourd'hut, Québec, Presses de l'Université Laval, 2007.

9. Martine-Emmanuelle LAPOINTE, «Présentacion*, http://www.litffa.umontreal.ca/personnel/LAPOINTE_Martine_ Emmanuelle.hrm (13 janvier 2008).

10. Suzanne CLAVETTE, Les dessous d'Asbestos. La lutte idéologigue contre la participation des travailleurs, Québec, Presses de P'Université Laval, 2005, p. 5. 
poésie, etc.), mais en s'attaquant cette fois au domaine religieux. En d'autres termes, il s'agit de reconnaître que l'Église catholique, définie jadis comme essentiellement conservatrice et autoritaire, n'a pas seulement servi à retarder "l'entrée du Québec dans la modernité", mais qu'elle a aussi joué, ici, un rôle plus ou moins "révolutionnaire».

Depuis au moins la parution de Sortir de la "Grande noirceur", en 2002 (essai qui reprend un article publié trois ans plus tôt dans le numéro spécial «Le Chainon manquant", de la revue Société), les travaux s'accumulent sur un tel sujet. La quatrième de couverture de l'essai de E.-Martin Meunier et Jean-Philippe Warren annonçait bien ses couleurs:

Nous serons polémistes à dire les choses telles que nous les concevons: le laïcisme et l'anticléricalisme farouches de la Révolution tranquille voilent l'essentiel, à savoir que celle-ci consacre, pour une large part, une sortie religieuse de la religion".

Cette perspective, que ni Meunier ni Warren ne peut se vanter d'avoir proposée le premier (voir les publications de Gabriel Clément, Jacques Cousineau, Jean Hamelin ou Lucia Ferretti), a récemment fait florès. Il n'y a qu'à consulter la Bibliographie des ouvrages et des articles sur l'histoire religieuse du Québec et du Canada français ${ }^{2}$, réalisée par les infatigables Christine Hudon et Guy Laperrière, pour se rendre compte du dynamisme de ce secteur depuis au moins cinq ans. Parmi une série de titres, mentionnons seulement Quand la jeunesse entre en scène, de Louise Bienvenue ${ }^{13}$, pour la force et l'élégance de la démonstration. Cette auteure nous fait mieux connaître les voies empruntées, dans les années d'immédiat après-guerre, par une jeunesse catholique idéaliste et fervente, qui cherche à se réconcilier avec les thématiques progressistes (socialisme, loisirs, égalité des sexes, etc.) sans rompre son lien avec Rome.

Dans cette brève note critique, $j$ 'ai choisi quelques parutions qui me semblent illustrer de belle façon l'attention renouvelée des jeunes chercheurs pour l'Église. Il n'est nulle question ici de prétendre à quelque exhaustivité que ce soit. Comme je l'ai dit plus haut, mon but est plutôt de cerner un nœud de préoccupations, et de baliser une manière originale de

$$
+4
$$

11. E.-Martun MEUNIER et Jean-Philippe WARREN, Sortur de la grande noirceur: lhorizon personnalsse de la Rtvolution tranquille, Sillery, Septentrion, 2002.

12. Christıne HUDON et Guy LAPERRIÈRE, * Biblıographie des ouvrages et des articles sur l'historre religieuse du Québec et du Canada françass, http://www.cseq.uqtr.ca/schec/documents_pdf/bibliographie_schec_2007.pdf (20 janvier 2008). 13. Louse BIENVENUE, Quand la jeunesse entre en scène. L'Action catholtque avant la Revolution tranquille, Montréal, Boréal, 2003. 
penser le fait religieux. Il me semble néanmoins que Esdras Minville, Les dessous d'Asbestos, Échanges intellectuels entre la France et le Québec (19302000) et Lionel Groulx, le mythe du berger constituent des exemples stimulants, respectivement, du champ des biographies intellectuelles, des idéologies, des réseaux et des stratégies de distinction. Bien entendu, on aurait pu faire place au champ de l'histoire institutionnelle, comme on aurait pu discuter du champ des études littéraires ${ }^{14}$, mais il faut savoir, comme on dit, se limiter, d'autant plus que ces approches me paraissent, au pifomètre, moins populaires que les autres dans les départements d'histoire du Québec.

Du côté des biographies, le mémoire de maîtrise de Dominique Foisy-Geoffroy ${ }^{15}$, dirigé par Pierre Trépanier (qui est presque en train de faire école), offre un condensé de nombreux thèmes nodaux de la période de l'entre-deux-guerres. La division en chapitres ("notions fondamentales", "données du problème", "un projet de renouveau") demeure un peu académique, et l'exposition des idées de Minville paraît parfois mécanique. L'auteur évoque le fait que le catholicisme social dont Minville s'est fait le défenseur ne s'alimente pas seulement de lectures en bibliothèque, mais s'abreuve aussi à des frustrations vécues; toutefois, on aurait voulu connaître davantage les personnages de la pièce dans laquelle l'économiste de l'Université de Montréal a voulu jouer un rôle. On aurait voulu sentir davantage d'espoirs et de colère derrière le portrait du professeur. Qu'à cela ne tienne! Le propos de Foisy-Geoffroy reste toujours rigoureux, insistant avec raison sur l'ambition de totalisation qui inspire Minville et sur la fin spirituelle qu'il assigne aux réalités économiques et sociales. Chez lui, l'Église chapeaute la cité terrestre, et prime à la fois la société civile, l'État, la nation. Mieux, elle dicte le chemin à suivre, à travers l'enseignement formulé par les encycliques pontificales. C'est de l'intérieur même de la pensée catholique que Minville tente de répondre aux défis de son temps. Alors que Foisy-Geoffroy relie cette vision du monde à la sociologie le playsienne, il me semble, comme a tenté de le démontrer Nicole Gagnon, qu'il faudrait plutôt la situer dans le sillage de la sociologie doctrinale mise à l'honneur dans les institutions de diffusion de la pensée sociale, comme l'École sociale populaire ou les Semaines sociales. Cela aurait évité à l'auteur le piège de l'anachronisme, quand il parle du personnalisme de Minville, une étiquette qu'il vaut mieux réserver à la sociologie lavalloise (qui aura, à l'évidence, l'Université Laval

$$
+4+
$$

14. Lire, par exemple, Cécile VANDERPELEN-DIAGRE, Memoure d'y croire. Le monde catholique es La lutterature au Québec (1920-1960), Québec, Noca bene, 2007.

15. Dominique Forsy-GeOFfroY, op. cit. 
pour foyer, sans que cela ne l'empêche d'essaimer un peu partout, à Montréal comme ailleurs).

Le deuxième ouvrage considéré dans cette note critique est celui de Clavette, tiré de sa thèse de doctorat ${ }^{16}$. Le cœur de sa démonstration du dynamisme de la pensée catholique dans les années d'après-guerre, c'est la publication d'un petit document intitulé La participation des travailleurs à la vie de l'entreprise. Passant à côté de maints travaux importants liés à son sujet, et faisant comme si elle découvrait un terrain vierge (ou alors mal étudié: selon elle, Jacques Rouillard ne sait pas calculer, Gilles Bourque et Jules Duchastel confondent les catégories politiques, Michel Sarra-Bournet se trompe à répétition, Pierre Trépanier n'est pas un historien sérieux, Simon Lapointe est borné, etc.), l'auteure s'attache à montrer que l'idéal de la participation des travailleurs à l'entreprise représente quelque chose de majeur dans les années d'après-guerre (oubliant, pour justifier son enthousiasme, de comparer cet idéal avec celui du corporatisme et du coopératisme, ou même de la colonisation). La lutte autour de cette panacée aux maux des ouvriers (bien supérieure, semble-t-il, à l'impôt) est décrite comme un combat entre progressistes et conservateurs. Les chapitres laissent place à peu d'ambiguïté: "Sous le signe de la libération", "La lutte idéologique s'embrase", "La bataille fait rage", "Victoire des forces conservatrices". Gérard Dion est le véritable héros de cette histoire hagiographique (l'auteure parle "d'événements épiques»!) : sa revue (le Bulletin des relations industrielles), apprend-on, est le lieu de plus intense diffusion de l'idéal participationniste et le Département des relations industrielles de l'Université Laval (dirigé par Gérard Dion) est «le centre névralgique de ce courant de pensée». On aura droit, comme multiples preuves à conviction, aux articles de Gérard Dion, aux lettres de Gérard Dion, aux interventions publiques de Gérard Dion, voire aux notes de cours de Gérard Dion. À la lecture même de la thèse, il appert pourtant que les travailleurs, loin de réclamer d'abord la participation à l'entreprise, souhaitent des augmentations salariales et de meilleures conditions de travail, tout simplement, et que les patrons en sont encore à refuser l'atelier fermé et la formule Rand. Une poignée d'intellectuels marginaux parlent bel et bien de cogestion, mais ils sont écoutés d'une oreille plutôt distraite. Quand aux patrons, ils aiment faire grand cas de quelques déclarations isolées de certains aumôniers plus syndicalistes, afin de brandir la menace du communisme et d'éviter de débattre des récla-

$$
+4+
$$

16. Suzanne ClaveTTE, op. ctt. 
mations concrètes. D'ailleurs, tout progressistes que soient les catholiques rangés derrière Dion, Clavette aurait dû reconnaître que leur idéologie a abouti à une impasse et que plus personne ne revendique réellement aujourd'hui ce genre de réforme de l'entreprise.

L'étude des réseaux est un autre champ à la mode dans les départements d'histoire. Stéphanie Angers et Gérard Fabre veulent montrer les ponts qui relient le Québec et la France, et pour cela utilisent comme "révélateur" les contacts ayant existé entre la revue Esprit et les revues La Relève, Cité libre, Parti pris et Possibles ${ }^{17}$. Alors que, dans le cas de Cité libre, la comparaison va de soi (cette revue se voulant une sorte d'Esprit québécois, de l'aveu même de ses animateurs), le choix des trois autres peut paraître arbitraire, à moins de postuler que ces revues sont réellement les plus déterminantes dans l'histoire québécoise. Pourquoi, en effet, ne pas avoir analysé les liens avec L'Action française, Relations, Liberté ou Maintenant? Toujours est-il que, la sélection faite, il est possible de lire les influences françaises sur le cheminement des collaborateurs québécois. Maritain est décrit comme le "guide incontesté " de La Relève, Mounier comme le «maître» de Cité libre. En revanche, dans les années soixante, la chrétienté québécoise se fragmente. Les maîtres à penser de la jeunesse sont de moins en moins issus du sérail catholique. Jean-Paul Sartre est la figure tutélaire de Parti pris. Dans les derniers chapitres de leur ouvrage, on dirait, pour cette raison, que Fabre et Angers étirent la sauce, faisant grand cas du fait que Jean-Marie Domenach résume un article de Trudeau dans les pages d'Esprit, ou que Gabriel Gagnon y fasse paraître deux articles sur le second référendum de 1995. Les réseaux deviennent bien minces pour prétendre à une influence profonde et durable. Aussi, on aurait voulu en savoir davantage sur la nature personnaliste du fédéralisme des citélibristes, sur les interprétations divergentes de la philosophie d'Esprit selon les collaborateurs québécois (le nom de Fernand Dumont côtoie mal celui de Trudeau, même dans les années cinquante), sur la conception fonctionnelle ou charnelle de la nation chez les uns et les autres (tout le monde ne sera pas convaincu par la lecture républicaine que Fabre et Angers font du personnalisme de Domenach). Cette première tentative de baliser le territoire des échanges intellectuels est toutefois, au total, stimulante. La religion y trouve une place, puisque c'est le

$$
4+4
$$

17. Stéphanie ANGERS et Gérard FABRE, Echanges intellecruels entre la France et le Québec, 1930-2000. Les reseaux de la revue Espric avec la Relève, Cité lıbre, Parti pris et Possıbles, Paris/Québec, L'Harmatran/Presses de l'Université Laval, 2004 . 
catholicisme qui, au-delà de la nationalité ou de la langue, unit La Relève, Cité libre et Esprit dans une même communauté de destin pendant l'entredeux-guerres. En revanche, une fois le nationalisme sécularisé devenu le centre des préoccupations québécoises, il est normal d'assister à un déclin de l'influence de Mounier dans les pages de Parti pris ou Possibles, au profit des penseurs de la décolonisation comme Fanon ou Berque.

Je termine par une critique de l'ouvrage de Marie-Pier Luneau ${ }^{18}$, l'un des meilleurs sans aucun doute de la récolte récente. Luneau aborde la question de la modernité de l'Église en interrogeant la posture militante du chanoine Lionel Groulx et les stratégies utilisées par ce dernier pour s'imposer auprès du grand public et des élites littéraires. Le portrait qu'elle dégage est extraordinaire, car il évite les catégories de "conservateurs» et de "progressistes" qui peuvent brouiller l'analyse. Il s'agit seulement de comprendre comment le chanoine Groulx est devenu une référence incontournable au Canada français, et si ce mérite tient seulement aux qualités intrinsèques de son ouvre ou aussi à des stratégies de promotion individuelle. En effet, jouant le rôle de celui qui ne cède aux louanges qu'à contrecœur et ne renonce jamais à l'humilité, dont il prétend avoir fait vœu, que sous de puissantes pressions extérieures, Groulx met sans cesse en scène un récit providentialiste qui l'absout de la célébrité. Ce n'est point lui, affirme-t-il dans ses mémoires, qui choisit le succès: la réussite lui vient sans que jamais il ne la réclame. L’analyse détaillée de Luneau dévoile pourtant les moyens dont use l'auteur de L'appel de la race pour occuper une place centrale dans le champ intellectuel : il n'hésite pas à multiplier les pseudonymes afin d'imprimer des articles qui le portent aux nues et célèbrent en lui un maître de la plume. Les deux tiers de ses textes publiés sous un nom d'emprunt dans L'Action française parlent de lui-même ou de ses livres! Comme quoi, en une époque où la critique littéraire n'existe pas encore vraiment et où les éditeurs n'assurent pas la publicité de leur catalogue, on n'est jamais aussi bien servi que par soi-même. Et puis, au fond, comment un auteur qui défend une conception aussi farouchement engagée de l'écrivain aurait-il pu s'abstenir de s'engager pour la promotion de son œuvre nationale? En servant ses fins, il est certain que Groulx a la conviction intime de servir son peuple.

$$
+4
$$

18. Marie-Pier LUNEaU, Lionel Groulx. Le mythe du berger, Montréal, Leméac, 2003. 


\section{UN CHAMP PROMETTEUR}

Les quatre approches décrites ici suivent une sorte de gradation vers une échappée du religieux. Foisy-Geoffroy est celui qui enracine le plus son personnage dans le terreau catholique de son époque:

Si on prend une perspective globale, on n'hésitera pas à situer Minville d'abord et avant tout dans le grand courant de la pensée catholique sociale qui a pris corps depuis le pontificat de Léon XIII, une pensée qui doit d'ailleurs beaucoup à l'inspiration thomiste ${ }^{19}$.

Dans le cas de Clavette, il se trouve que son personnage central - Gérard Dion - est un prêtre, mais cela apparaît presque superfétatoire. Elle parle d'abord du combat en faveur des idées progressistes, pas directement du clergé. Angers et Fabre s'intéressent encore moins au catholicisme. Leur objectif est de dégager les canaux à travers lesquels les idées circulent, laissant de côté l'analyse en profondeur du contenu de ces idées. L'ouvrage nous en apprend énormément sur une foule de choses, et d'abord sur les logiques culturelles des appropriations réciproques, mais point sur l'Église. Enfin, dans le cas de Luneau, on pourra presque s'étonner de retrouver sa thèse dans une note critique qui porte sur les ouvrages d'histoire du catholicisme canadien-français, tant le religieux n'y est nulle part une catégorie centrale. Groulx est traité comme un auteur sans confession particulière. Sa qualité de chanoine n'y est jamais invoquée comme déterminante ou même notable. Je pense pourtant que le livre de Luneau a sa place dans la bibliographie des ouvrages d'histoire du catholicisme canadien-français: il trace la ligne limite (en même temps, affirmeront certains, qu'idéale) où la religion devient un phénomène social comme un autre, reçu seulement dans sa phénoménologie, en dehors de ses motivations morales ou spirituelles intrinsèques. Pour des jeunes qui n'ont pas reçu une socialisation religieuse, on pourrait craindre qu'il s'agisse là de la seule voie interprétative à suivre: les études religieuses se poursuivraient alors, en quelque sorte, en l'absence du religieux.

Les quatre études décrites ici montrent bien la place, le rôle et les stratégies des catholiques dans l'entre-deux-guerres. Chacun dégage à sa façon ce que ces hommes et ces femmes avaient de "moderne». Clavetre fait de l'abbé Dion un progressiste, voire un révolutionnaire en guerre contre les forces obscurantistes qui menacent de submerger le Québec. Luneau dévoile l'efficacité des procédés utilisés par un clerc pour s'imposer dans le champ

$$
+4+
$$

19. Dominique FoISY-GeOFFROY, op. cit., p. 24. 
littéraire. Angers et Fabre démontrent que La Relève ou Cité libre se tenaient au diapason de ce dont discutaient en France les cercles les plus éclairés et les plus avant-gardistes. Enfin, Dominique Foisy-Geoffroy vise à montrer à quel point «la pensée traditionaliste n'est pas restée passive [...] face aux défis posés par la modernisation de la société: elle avait aussi un projet de société, des suggestions de réformes, un ordre de valeurs à proposer aux Canadiens français». Chez la plupart, la perspective adoptée s'éloigne d'une téléologie qui conduirait des Anciens aux Modernes, c'est-à-dire de Lionel Groulx à... Gérard Bouchard! Et pour cause. S'avouant déçus de l'évolution de la société québécoise, ils n'hésitent pas à reprocher à leurs devanciers immédiats une certaine ingratitude. Pascale Ryan n'hésite pas à invoquer "une réforme de l'éducation mal menée", "un système de santé dépersonnalisé et débordé» et "des finances publiques dans un état lamentable». Xavier Gélinas souligne, pour sa part, les "réveils douloureux" ayant suivi les réformes échevelées des années soixante. Chacun fera ce qu'il veut de ces prises de positions, plus politiques que scientifiques. Il demeure que, refusant de succomber à la vision angélique du devenir québécois depuis longtemps dénoncée par Jocelyn Létourneau (dans des textes qui, parfois, ne manquent pas euxmêmes d'angélisme), une palette de jeunes chercheurs caressent l'ambition de comprendre le Canada français dans sa dynamique propre, comme une totalité autonome, sans tenter de transformer ce passé en simple faire-valoir d'une contemporanéité dorée. Peu importe la justesse des raisons qui ont mené à la redécouverte du Canada français comme fait anthropologique, cette posture paraît heureuse et prometteuse. Espérons que, quelles que soient leurs motivations profondes, les étudiants de maîtrise et de doctorat continueront sur cette voie, déjà bellement balisée par les quelques études récentes qui meublent les tablettes des bibliothèques. 